\title{
ENHANCEMENT OF THE DOUBLE FLEXIBLE PACE SEARCH THRESHOLD DETERMINATION FOR CHANGE VECTOR ANALYSIS
}

\author{
S. A. Azzouzi ${ }^{\mathrm{a}, \mathrm{b},},{ }^{*}$ A. Vidal ${ }^{\mathrm{a}}$, H. A. Bentounes ${ }^{\mathrm{b}}$ \\ ${ }^{\mathrm{a}}$ Instituto de Telecomunicaciones y Aplicaciones Multimedia (iTEAM), Universitat Politècnica de València, \\ Valencia, Spain - azzouzi.soufiane@hotmail.com, avidal@dcom.upv.es \\ ${ }^{\mathrm{b}}$ Component, signal and systems laboratory, University Abdelhamid Ibn Badis of Mostaganem, \\ Mostaganem, Algeria - bhadjadda@gmail.com
}

KEY WORDS: Threshold determination, Change Detection, Change Vector Analysis (CVA), Supervised Classification, Land Cover Land Change.

\begin{abstract}
:
Remote sensing is one of the most reliable ways to monitor land use and land cover change of large areas. On the other hand, satellite images from different agencies are becoming accessible due to the new user dissemination policies. For that reason, interpretation of remotely sensed data in a spatiotemporal context is becoming a valuable research topic. In the present day, a map of change has a great significant for scientific purposes or planning and management applications. However, it is difficult to extract useful visual information from the large collection of available satellite images. For that reason, automatic or semi-automatic exploration is needed. One of the key stages in the change detection methods is threshold selection. This threshold determination problem has been addressed by several recent techniques based on Change Vector Analysis (CVA). Thus, this work provides a simple semi-automatic procedure that defines the change/no change condition and a comparative study will be involved together with the previous existing method called Double Flexible Pace Search (DFPS). This study uses Landsat Thematic Mapper scenes acquired on different dates in an Algerian region. First, some training data sets containing all possible classes of change are required and their respective supervised posterior probability maps for each scene are obtained. The selected supervised classifier is based on the Maximum Likelihood method. Then four training sets (two sets from each date) are chosen from their corresponding probability maps based on their spatial location in the original images. The optimal average will be obtained as an average of the thresholds obtained at every set. This work verifies that the proposed approach is effective on the selected area, providing improved change map results.
\end{abstract}

\section{INTRODUCTION}

Satellite data contain very useful information that can provide spatiotemporal changes in land cover/ land use. Different change detection techniques were used in the past to define the cover changes as resumed in (Lu et al., 2004). Change vector analysis (CVA) is a very effective method used in the process of extracting, analyzing and defining the change information from the satellite data, as described in (Bovolo and Bruzzone, 2007), (Malila, 1980) and (Xian. et al., 2009). CVA can present as output data the total magnitude of change and the angle of change direction between two different time samples from multispectral satellite images as shown in (Collins and Woodcock, 1994), (Jensen, 1995) and (Johnson and Kasischke, 1998).

In previous works, several automatic and semiautomatic methods were developed to search the threshold of change magnitude. The threshold selection is a key stage in CVA that has deserved further investigation in (Ding et al., 1998), (Johnson and Kasischke, 1998) and (Smits and Annoni, 2000). In this work, a semiautomatic method called Double-Window Flexible Pace Search (DFPS) (Chen et al., 2003) was used as a starting point. Then, a modification of this method is proposed. The main difference in the employment of four samples from the two images. This new methodology is explained and the corresponding results are presented in this communication.

Finally, a comparison and complexity evaluation between the original and the modified method will be done. The Success Percentage as in (Chen et al., 2003) will be the tool used to define the optimal threshold selection.

${ }^{*}$ Corresponding author. This is useful to know for communication with the appropriate person in cases with more than one author

\begin{tabular}{cccc}
\hline Dates & Landsat ID N $^{\circ}$ & Instrument & Path/Row \\
\hline 14-06-2002 & TM 5 & Bumper & $196 / 035$ \\
04-06-2010 & TM 5 & Bumper & $196 / 035$ \\
\hline
\end{tabular}

Table 1: Characteristics of the studied area.

\section{METHODOLOGY}

\subsection{Study area}

The selected area have been acquired from the Landsat 5 program by the Bumper instrument. It is available in the (USGS) archive (USGS, 2014).

The study area in Blida, Algeria is located in north central region of Africa as shown in Fig. 1. Two Landsat images acquired respectively on June 14, 2002 and June 4, 2010, shown in Fig. 2, were employed in this study. It presents a total surface equal to $788.18 \mathrm{~km}^{2}$, located at a latitude of $36.34^{\circ}$ (North) to $36.13^{\circ}$ (North) and longitude of $1.98^{\circ}$ (East) to $2.36^{\circ}$ (East). In this study, a sub-area of $1130 \times 775$ pixels is chosen for further analysis.

\subsection{Image pre-processing}

In Remote Sensing, energy reflected by Earth surface is represented by a Digital Number that depends on the fraction of incoming solar radiation value, the surface slope and its orientation, the possible surface anisotropy, and the variable atmospheric components as described in (Srinivasulu and Kulkarni, 2004). It obviously also depends on the instantaneous state of the land surface. Therefore, radiometric normalization is required for correcting all possible pixel spectral changes caused by the following factors: 


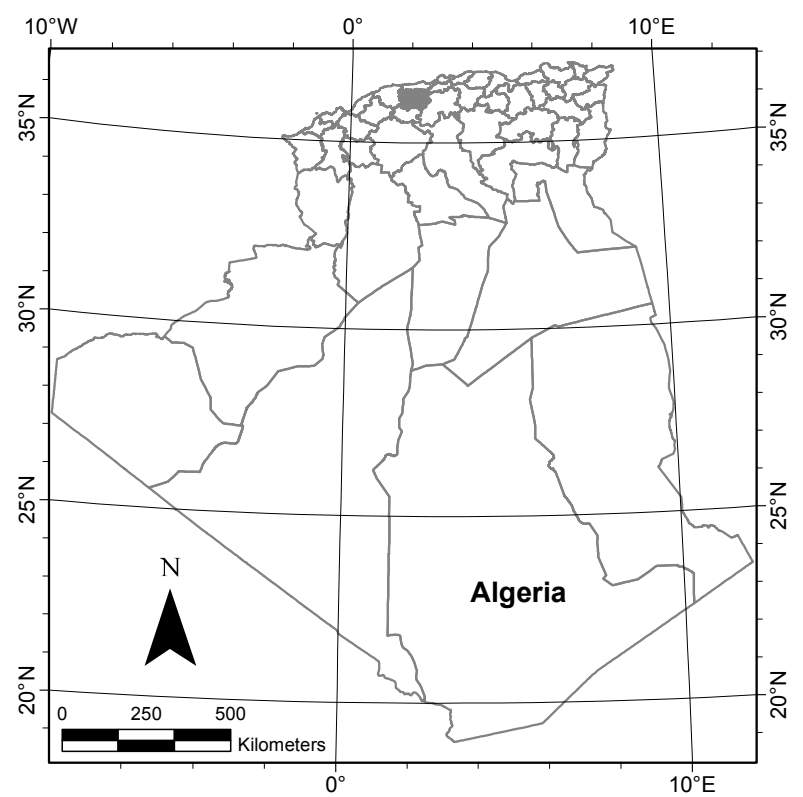

Figure 1: Map localisation of the studied area of Algeria.

a) differences between the images due to changes in illumination values, variation in Sun and Earth distance, solar azimuth or zenith angle, b) changing atmospheric conditions causing different scattering and absorption, c) sensor differences, d) soil moisture and e) vegetation phenology. Some of these factors must be corrected in order to make sure that the obtained changes are only caused by surface changes. The selected radiometric correction is based on finding permanent image features, that can also help for image co-register.

Geometric correction and co-registration is also needed as described in (Xiangyang. et al., 2010). The geometric correction and co-register provides a unique grid in a common coordinate system for both images. After geometric rectification and image co-registration provided by the ENVI tools using 100 control points, the Root Mean Square Error (RMSE) between the images in Fig. 2 was approximately equal to 0.11 pixels.

\subsection{Change Vector Analysis}

The general concept of change detection technique based on dynamic changes in the multispectral space using CVA was presented in (Malila, 1980). The CVA method employs the angle of change and the magnitude of change from time $t_{1}$ to time $t_{2}$ as shown in (Johnson and Kasischke, 1998) and (Lu et al., 2004).

In this paper, we have implemented the change vector analysis in posterior probability space. The posterior probability vectors were obtained from the supervised Maximum Likelihood Classifier (MLC) classifier using five pre-defined classes: bare soil, dense vegetation, no-dense vegetation, urban and water areas. All bands from Landsat $5 \mathrm{TM}$ sensor were used except from the thermal infrared one. The MLC technique provides two Posterior Probability Maps, each one for each date of acquisition:

$$
\begin{aligned}
P^{m} & =\left(p_{1}^{m}, p_{2}^{m}, \ldots, p_{c}^{m}\right), \\
P^{n} & =\left(p_{1}^{n}, p_{2}^{n}, \ldots, p_{c}^{n}\right),
\end{aligned}
$$

where $P^{m}$ and $P^{n}$ represent the posterior probability map at time $t_{1}$ and $t_{2}$, respectively. Index $1,2, \ldots, c$ denotes the class involved.

The relationship between the corresponding pixel pair of posterior probability maps can be characterized by a change vector.

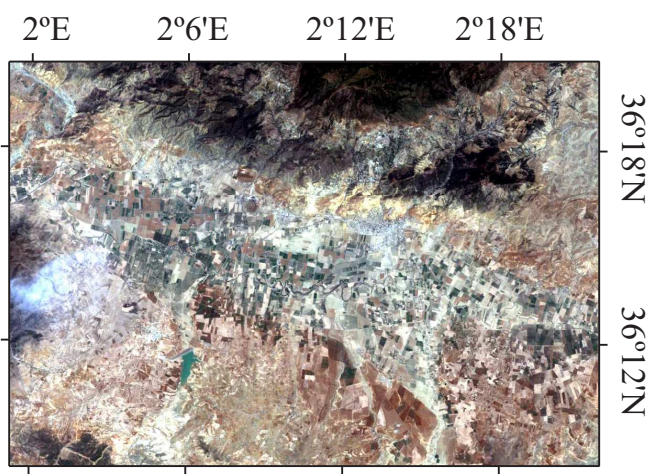

(a)

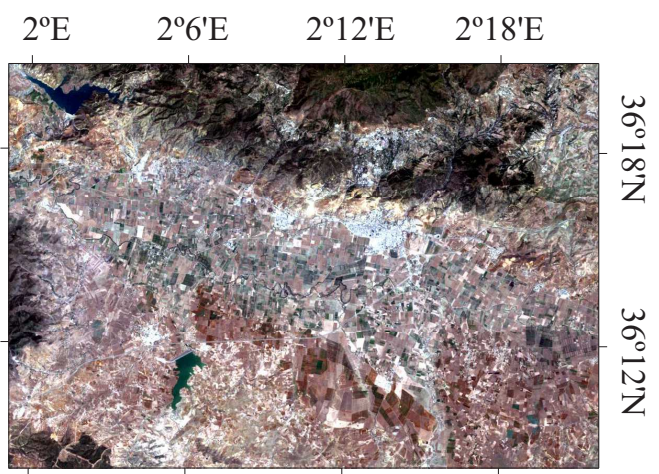

(b)

Figure 2: The studied area of Algeria: image acquired on June 14, 2002 in a), and image acquired on June 04, 2010 in b).

The change vector of the posterior probability space can be represented as follows:

$$
\Delta P=P^{n}-P^{m},
$$

where $\Delta P$ is the posterior probability difference. It contains all the change information between the images taken at time $t_{1}$ and time $t_{2}$.

The magnitude of $\Delta P$ is given by:

$$
\|\Delta P\|=\sqrt{\left(p_{1}^{n}-p_{1}^{m}\right)^{2}+\ldots+\left(p_{c}^{n}-p_{c}^{m}\right)^{2}}
$$

In this context, a large value for $\|\Delta P\|$ indicates a high possibility of change pixel. However, if a hard decision (change/no change) has to be taken, then a threshold selection is a very critical step in the procedure. Figure 3 shows this procedure, where the optimal threshold selection step is the stage that will be further analyzed.

\subsection{The Double-Window Flexible Pace threshold selection}

The original DFPS described in (Chen et al., 2003) calculates the optimal threshold to identify the changed and unchanged pixels in the change magnitude with the evaluation of the best final success percentage. It is based on the selection of the threshold from one training sample in the study area. A threshold related to the maximum accuracy of change detection in the training samples will be considered as optimal threshold for the DFPS method.

The key step is how to get this assured threshold. In the present paper, an enhancement has been made to DFPS method to achieve the highest accuracy. 


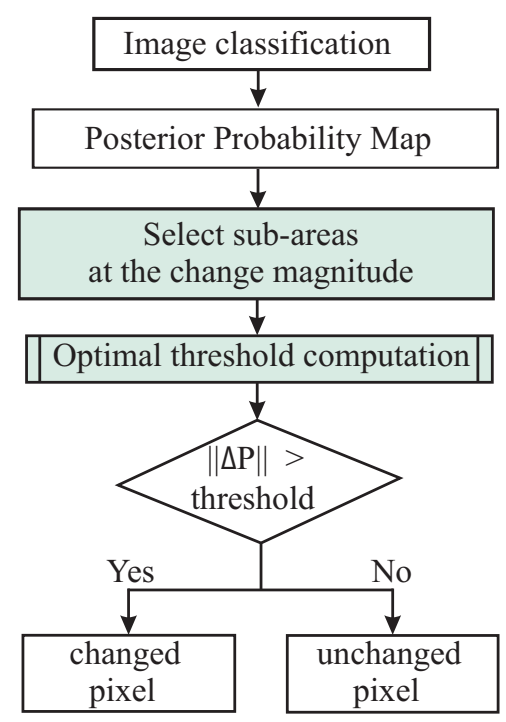

Figure 3: Flowchart of the CVA change detection.

\subsection{The proposed Algorithm for threshold selection}

In this work, a modified algorithm for threshold selection is proposed. Therefore, the key of this section is to get the adequate threshold value. Figure. 4 shows the selected flowchart that provides an optimum threshold. We select four typical training samples that contain all possible changes at different geographic locations in the image.

First, we select the appropriate training samples. Then, we compute $\|\Delta P\|$ for each pixels of the training set. We assume that the change magnitude $\|\Delta P\|$ is in the range $\left[\|\Delta P\|_{\min },\|\Delta P\|_{\max }\right]$ for each training set. The flow plan is as follow:

1. Choose the precision value, which is a parameter that stops the iteration process as in (Chen et al., 2003).

2. Calculate the maximum $\left(\|\Delta P\|_{\max }\right)$, minimum $\left(\|\Delta P\|_{\min }\right)$, and the mean $\left(\|\Delta P\|_{\text {avg }}\right)$ of each training sample separately.

3. The initial pace is taken as $\|\Delta P\|_{\text {avg }}$.

4. If the precision condition is not satisfied, then a new search process begins in the same interval with the pace value taken as $\|\Delta P\|_{\text {avg }} / 2,\|\Delta P\|_{\text {avg }} / 4, \ldots$.

5. The optimal threshold value will depend on the highest Kappa coefficient and a reasonable demanded accuracy in the search process using the success rate defined in (Chen et al., 2003).

The final number of iterations obviously increases with the demanded accuracy. This semi-automatic technique gets the optimal threshold to identify land cover change. As an advantage, this method controls the final precision of the search process, since it balances commission and omission errors. This optimal value can change according to the training samples and to the desired success rate.

\section{RESULTS}

The Double-Window Flexible Pace Search is based on searching the threshold by comparing both images within a sample training data set at different times $t_{1}$ and $t_{2}$. The search process began in the range $[0-6.4031]$ with the pace of 0.6403 at the first step. The optimal threshold is related to the success rate percentage between the maximum and the minimum value was less than 0.05

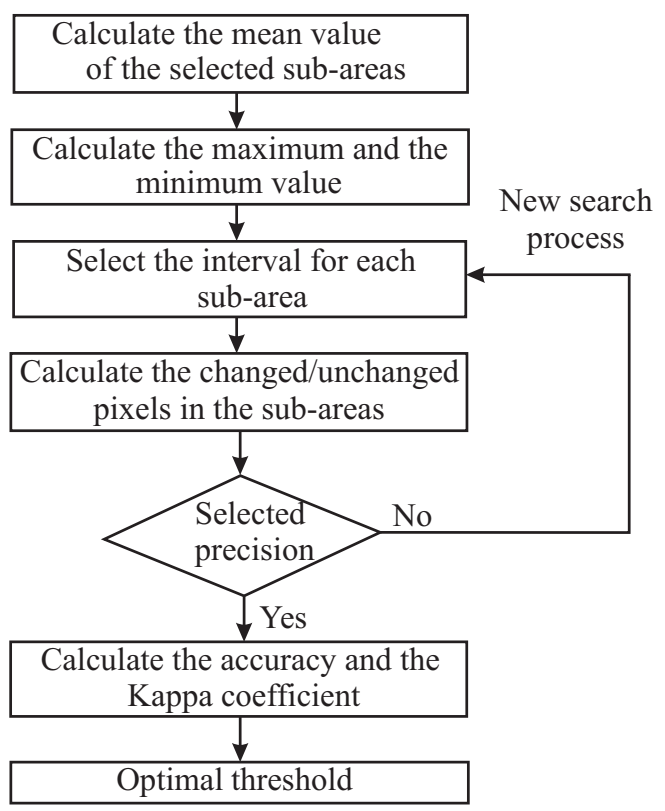

Figure 4: Flowchart of the proposed threshold determination algorithm.

percent of each iteration at this process as described in (Chen et al., 2003).

As a result, the threshold of change magnitude was obtained as the value 3.2015 with a success percentage of $64.26 \%$. Table 2 presents the progress of the search process in the previous range with the paces $0.6403,0.2134,0.0711$, and 0.0355 . The process of search needed 31 iterations. The optimal threshold to decide if a pixel was changed or not in the studied area was 3.2015 using DFPS.

The proposed method needs the selection of four different spatial localization samples as first step. The search process was based on the four training samples at the change magnitude.

As the four samples training contains all types of change, the computing of the algorithm parameters was done within the range [0 - 6.4031], with the paces of $2.1425,1.2667,1.5558,0.4796$, for the first, second, third, and the fourth samples training, respectively. The selection of the best optimal threshold was done by the percentage of the success rate in the iteration process following the success rate definition in (Chen et al., 2003).

As a result, the best value of threshold was obtained 3.1116 in the third set with a success percentage of $93.959 \%$. The progress of the searching process is shown in the Table 3 for all the selected training samples. It contains 2, 5, 4 and 13 iterations for the first, second, third, and the fourth selected training samples, respectively.

In order to compare the performance of both methods, Table 4 presents the accuracy results obtained in the selected sub area with for each threshold value. A Kappa coefficient of 0.864 and 0.865 with an overall accuracy of $93.962 \%$, and $93.959 \%$ were achieved for the DFPS and the proposed algorithm, respectively. The accuracy of change was estimated using validation data.

\begin{tabular}{|c|c|c|}
\hline Method & Overall Accuracy & Kappa Coefficient \\
\hline DFPS & $93.962 \%$ & 0.864 \\
\hline Algorithm & $93.959 \%$ & 0.865 \\
\hline
\end{tabular}

Table 4: Accuracy assessment of the methods 


\begin{tabular}{|c|c|c|c|c|c|c|c|}
\hline \multicolumn{2}{|c|}{$\begin{array}{c}\text { Range }=6.4031-0 \\
\text { Pace }=0.6403\end{array}$} & \multicolumn{2}{|c|}{$\begin{array}{c}\text { Range }=3.8418-2.5612 \\
\text { Pace }=0.2134\end{array}$} & \multicolumn{2}{|c|}{$\begin{array}{c}\text { Range }=3.4149-2.9881 \\
\text { Pace }=0.0711\end{array}$} & \multicolumn{2}{|c|}{$\begin{array}{c}\text { Range }=3.2726-3.1304 \\
\text { Pace }=0.0355\end{array}$} \\
\hline Threshold & $\begin{array}{c}\text { Success } \\
\text { Percentage }\end{array}$ & Threshold & $\begin{array}{c}\text { Success } \\
\text { Percentage }\end{array}$ & Threshold & $\begin{array}{c}\text { Success } \\
\text { Percentage }\end{array}$ & Threshold & $\begin{array}{c}\text { Success } \\
\text { Percentage }\end{array}$ \\
\hline 6.4031 & $30.07 \%$ & 3.8418 & $59.13 \%$ & 3.4149 & $62.98 \%$ & 3.2726 & $64.12 \%$ \\
\hline 5.7627 & $39.17 \%$ & 3.6284 & $61.73 \%$ & 3.3438 & $63.36 \%$ & 3.2371 & $64.20 \%$ \\
\hline 5.1224 & $43.39 \%$ & 3.4149 & $62.98 \%$ & 3.2726 & $64.12 \%$ & 3.2015 & $64.26 \%$ \\
\hline 4.4821 & $51.28 \%$ & 3.2015 & $64.26 \%$ & 3.2015 & $64.26 \%$ & 3.1659 & $64.22 \%$ \\
\hline 3.8418 & $59.13 \%$ & 2.9881 & $63.54 \%$ & 3.1304 & $64.17 \%$ & 3.1659 & $64.22 \%$ \\
\hline 3.2015 & $64.26 \%$ & 2.7746 & $62.63 \%$ & 3.0592 & $64.02 \%$ & 3.1304 & $64.17 \%$ \\
\hline 2.5612 & $61.10 \%$ & 2.5612 & $61.10 \%$ & 2.9881 & $63.54 \%$ & & \\
\hline 1.9209 & $56.96 \%$ & & & & & & \\
\hline 1.2806 & $60.28 \%$ & & & & & & \\
\hline 0.6403 & $11.13 \%$ & & & & & & \\
\hline 0.000 & $8.27 \%$ & & & & & & \\
\hline
\end{tabular}

Table 2: Results of threshold selection with the Double-window Flexible Pace Search (DFPS).

\begin{tabular}{|c|c|c|c|c|c|c|c|}
\hline \multicolumn{2}{|c|}{$\begin{array}{c}\text { First set } \\
\text { Pace }=2.1425\end{array}$} & \multicolumn{2}{|c|}{$\begin{array}{c}\text { Second set } \\
\text { Pace }=1.2667\end{array}$} & \multicolumn{2}{|c|}{$\begin{array}{c}\text { Third set } \\
\text { Pace }=1.5558\end{array}$} & \multicolumn{2}{|c|}{$\begin{array}{c}\text { Fourth set } \\
\text { Pace }=0.4796\end{array}$} \\
\hline Threshold & $\begin{array}{c}\text { Success } \\
\text { Percentage }\end{array}$ & Threshold & $\begin{array}{c}\text { Success } \\
\text { Percentage }\end{array}$ & Threshold & $\begin{array}{c}\text { Success } \\
\text { Percentage }\end{array}$ & Threshold & $\begin{array}{c}\text { Success } \\
\text { Percentage }\end{array}$ \\
\hline 2.1425 & $67.543 \%$ & 1.2667 & $55.947 \%$ & 1.5558 & $57.832 \%$ & 0.4796 & $42.781 \%$ \\
\hline 4.2849 & $71.282 \%$ & 2.5334 & $70.021 \%$ & 3.1116 & $\mathbf{9 3 . 9 5 9} \%$ & 0.9592 & $53.327 \%$ \\
\hline & & 3.8001 & $89.261 \%$ & 4.6673 & $66.481 \%$ & 1.4388 & $56.452 \%$ \\
\hline & & 5.0669 & $60.901 \%$ & 6.2231 & $48.724 \%$ & 1.9184 & $64.725 \%$ \\
\hline & & 6.3336 & $45.027 \%$ & & & 2.3980 & $68.432 \%$ \\
\hline & & & & & & 2.8776 & $72.329 \%$ \\
\hline & & & & & & 3.3572 & $92.027 \%$ \\
\hline & & & & & & 3.8368 & $89.084 \%$ \\
\hline & & & & & & 4.3163 & $69.623 \%$ \\
\hline & & & & & & 4.7959 & $66.209 \%$ \\
\hline & & & & & & 5.2755 & $58.431 \%$ \\
\hline & & & & & & 5.7551 & $52.739 \%$ \\
\hline & & & & & & 6.2347 & $48.204 \%$ \\
\hline
\end{tabular}

Table 3: Results of threshold selection with the proposed algorithm.

These results indicate that the proposed method is effective for searching the threshold value as the most important step in the change detection algorithm.

\section{DISCUSSION AND CONCLUSION}

The present paper presents a modified threshold selection for the CVA algorithm. The proposed method got a Kappa coefficient of 0.865 and an Overall Accuracy equal to $93.959 \%$. The results show that the proposed algorithm is efficient to determine the optimal threshold for change detection methods based on CVA using a lower number of iterations in the algorithm.

On the other hand, some other aspects like the correct training data selection and the correction of data are very critical in this process of threshold determination. The training samples and their correct classification play also an important role in the overall process. The proposed algorithm presents successful results and it can be an alternative to the original DPFS method.

\section{ACKNOWLEDGEMENTS}

This research has been done in the frame of EMMAG funding.

\section{REFERENCES}

Bovolo, F. and Bruzzone, L., 2007. A theoretical framework for unsupervised change detection based on change vector analysis in the polar domain. Geoscience and Remote Sensing, IEEE Transactions on 45(1), pp. 218-236.

Chen, J., Gong, P., He, C., Pu, R. and Shi, P., 2003. Landuse/land-cover change detection using improved change-vector analysis. In: Photogrammetric Engineering and Remote Sensing, Vol. 69, ASPRS, pp. 369-379.

Collins, J. and Woodcock, C., 1994. Change detection using the Gramm-Schmidt transformation applied to mapping forest mortality. Remote Sensing of Environment 50(3), pp. 267-279.

Ding, Y., Elvidge, C. and Lunetta, R. S., 1998. Survey of multispectral methods for land cover change detection analysis,. Sleeping Bear Press, New York, N.Y, USA.

Jensen, J. R., 1995. Introductory Digital Image Processing: A Remote Sensing Perspective. 2 edn, Prentice Hall PTR, Upper Saddle River, NJ, USA.

Johnson, R. D. and Kasischke, E. S., 1998. Change vector analysis: A technique for the multispectral monitoring of land cover and condition. International Journal of Remote Sensing 19(3), pp. $411-426$. 
Lu, D., Mausel, P., Brondzio, E. and Moran, E., 2004. Change detection techniques. International Journal of Remote Sensing 25(12), pp. 2365-2401.

Malila, W., 1980. Change vector analysis: An approach for detecting forest changes with landsat. In: Proceedings of Sixth Annual Symposium on Machine Processing of Remotely Sensed Data: Soil Information Systems and Remote Sensing and Soil Survey, IEEE, pp. 326-335.

Smits, P. and Annoni, A., 2000. Toward specification-driven change detection. Geoscience and Remote Sensing, IEEE Transactions on 38(3), pp. 1484-1488.

Srinivasulu, J. and Kulkarni, A., 2004. Estimation of spectral reflectance of snow from IRS-1D LISS-III sensor over the himalayan terrain. Journal of Earth System Science 113(1), pp. 117-128.

USGS, 2014. GLOVIS http://glovis.usgs.gov/.

Xian., G., Homer., C. and Fry, J., 2009. Updating the 2001 national land cover database land cover classification to 2006 by using landsat imagery change detection methods. Remote Sensing of Environment 113(6), pp. 1133-1147.

Xiangyang., S., Conggui., L. and Yizhen, S., 2010. Comparison and analysis research on geometric correction of remote sensing images. In: Image Analysis and Signal Processing (IASP), 2010 International Conference on, pp. 169-175. 